\title{
Methicillin Resistant Staphylococcus aureus (MRSA): An Interim Report of Carriage and Conversion Rates in Nursing Students
}

\section{RODNEY E. ROHDE, CHERYL ROWDER, TOM PATTERSON, GERALD REDWINE, BOB VÁSQUEZ, EMILLIO CARRANCO}

OBJECTIVE: To evaluate and characterize MRSA and staphylococci carriage and conversion rates in nursing students across clinical semester rotations and to describe risk factors.

DESIGN: A prospective, longitudinal cohort design (interim report) with three times of measurement. Data collected between August 2010 and May 2011 (ongoing longitudinal study to May 2012). Institutional Review Board approval (2010F5693).

SETTING: Texas State University, San Marcos, TX.

PARTICIPANTS: Eighty-seven nursing students.

INTERVENTIONS: A positive MRSA swab represented an end-point for a participant. Intervention offered was bactroban (mupirocin) for nasal decolonization and an oral antibiotic, doxycycline; posttreatment collection to verify decolonization prior to next clinical rotation.

MAIN OUTCOME MEASURES: Screening for Staphylococcus aureus and MRSA identification; confirmation and antibiotic susceptibility by Vitek 2 . Self-administered questionnaires collected demographics and risk factors. Generalized estimating equations calculated population-averaged panel logistic regression models allowing for an $\mathrm{AR}(1)$ error by Stata version 12.

RESULTS: MRSA colonization did not increase. S. aureus prevalence (20-26\%). Species prevalence other than S. aureus increased (9.2\% to $80 \%)$. The following associations were found to be statistically significant: boil or skin infection odds with $S$. aureus $(\mathrm{OR}=2.43$, $\mathrm{p}<.05)$, working or volunteering in healthcare facility odds with $S$. other $(\mathrm{OR}=2.72, p<.05)$ and gym and sports activities odds with $S$. other $(\mathrm{OR}=4.98, p<$ $.001)$.

CONCLUSIONS: MRSA colonization did not increase. Knowledge and understanding of MRSA (risks) may play a role in compliance and barrier precautions. S. aureus colonization remained stable (25$30 \%$ ). Species colonization other than S. aureus (e.g. S. epidermis, S. haemolyticus) increased to significant levels.

ABBREVIATIONS: MRSA $=$ Methicillin resistant Staphylococcus aureus; CA-MRSA=Communityassociated methicillin resistant Staphylococcus aureus; HA-MRSA=Healthcare-associated methicillin resistant Staphylococcus aureus; CLS = Clinical Laboratory Science; OR = Odds Ration; CI = Confidence Interval; HCWs = Healthcare Workers; Healthcare associated infections $=$ HAIs.

INDEX TERMS: Methicillin resistant Staphylococcus aureus, MRSA, Community acquired infections, Nursing research, Nosocomial infections, HAIs.

Clin Lab Sci 2012;25(2):94

Cheryl Rowder, PhD, RN, CCRC, Texas State University-San Marcos, San Marcos, TX

Tom Patterson, MS, BS, MT (ASCP), Texas State University-San Marcos, San Marcos, TX

Gerald Redwine, MS, MLS (ASCP), Texas State University-San Marcos, San Marcos, TX

Bob Vásquez, PhD, Texas State University-San Marcos, San Marcos, TX

Emillio Carranco, MD, Texas State University-San 
Marcos, San Marcos, TX

Rodney E. Rohde, PhD, MS, SV, SM(ASCP) ${ }^{C M} M B^{C M}$, Texas State University-San Marcos, San Marcos, TX

Address for Correspondence: Rodney E. Rohde, PhD, $M S, S V, S M(A S C P)^{C M} M B^{C M}$, Associate Professor and Associate Dean of Research, College of Health Professions, Texas State University-San Marcos, Clinical Laboratory Science, HPB 361, 601 University Drive, San Marcos, TX 78666-4616, 512-245-2562, rrohde@txstate.edu

\section{INTRODUCTION}

Healthcare associated infections have become one of the most costly and deadly growing public health threats of our time. The Centers for Disease Control and other studies estimate that Methicillin Resistant Staphylococcus aureus (MRSA) has surpassed HIV as the leading cause of morbidity and mortality in the U.S. ${ }^{1,2}$ For the past several decades, MRSA has become a serious problem in patients with exposure to the healthcare system, and is responsible for substantial morbidity and mortality in hospitals around the world. ${ }^{3}$ Today, healthcareassociated methicillin resistant Staphylococcus aureus (HA-MRSA) accounts for approximately $85 \%$ of all invasive MRSA infections. ${ }^{1}$

MRSA has also evolved in the community and is unrelated to the evolution of HA-MRSA in hospitals and other settings. These community associated strains, known as community-associated methicillin resistant Staphylococcus aureus (CA-MRSA) have been causing an increasing number of serious infections in nonhospitalized, previous healthy young people. ${ }^{4}$ CAMRSA is easily transmissible, not only between families, but also in larger close-contact communal settings such as prisons, schools, and sports team environments (e.g. locker rooms, wrestling mats, etc.). ${ }^{4,5}$ Environmental sources, such as the sharing of clothing, sports equipment, towels, razors, and soaps; improper care of skin trauma; crowded living conditions, along with lack of cleanliness and personal hygiene, are identified as possible risk factors for both CA-MRSA and HAMRSA infections. ${ }^{5-7}$ Regardless of the type of MRSA, the risk of MRSA carriage by healthcare workers (HCWs) poses a real threat to the healthcare environment as well as the general public.

Because nasal carriage (colonization) of $S$. aureus has been identified as a major risk factor for subsequent infections, an understanding of the risk factors for carriage of $S$. aureus and MRSA is crucial to understanding the potential for transmission and invasive infections. A variety of studies have examined community prevalence of nasal carriage in subpopulations including hospitals, outpatient settings, jails, and injection drug users. ${ }^{3,8-15}$ Several point prevalence studies have been conducted with medical students ${ }^{16-18}$ but very few, if any, longitudinal studies have examined the characteristics of $S$. aureus and MRSA in a population of HCWs. This project seeks to add to the knowledge of risk for acquisition as well as length of time to colonization of healthcare professionals.

The purpose of this research was to assess initial prevalence or acquisition of $S$. aureus or MRSA in a cohort of nursing students and to follow these students over five semesters of clinical care experiences. Hospitals have instituted specific contact precautions for patients who have a known positive nasal swab test for MRSA in the acute care setting. This care has not to this point included routine examination of healthcare workers for risk either to acquire this bacterium or to contribute to its spread. The question of screening for MRSA in HCWs remains understudied.

It was anticipated that the findings of this study would be utilized to (1) examine risk factors associated with conversion to MRSA colonization, (2) develop a future study examining prevalence of MRSA in healthcare workers from the public domain, (3) provide preliminary data to develop a pilot program to improve control and prevention of transmission of MRSA with respect to risk factors identified in this study, and (4) provide supportive data to institute an examination of compliance with contact isolation used in a local acute care setting including nursing attitudes toward enforcement, and patient comprehension of MRSA and contact isolation.

\section{MATERIALS AND METHODS}

\section{Sample and Data Acquisition}

A longitudinal (time-series) design was planned to determine the rate of $S$. aureus and MRSA carriage in an incoming cohort of nursing students and to describe exposures (risk factors) associated with carriage. Initial explanation of the study procedures was provided by the 
investigators from the School of Nursing. To reduce potential coercion, recruitment was accomplished by Clinical Laboratory Science (CLS) investigators. A purposive sampling strategy took place with the final sample consisting of nursing students over the age of eighteen. All participation was voluntary, and prior to participation, students authorized informed consent via the CLS personnel. The Institutional Review Board (IRB) of Texas State University-San Marcos approved all procedures and protocols for this study (\#2010F5693).

Investigators from two units of the College of Health Professions; School of Nursing and Clinical Laboratory Science (CLS) and the Student Health Center along with a statistician from the College of Applied Arts (Criminal Justice Department) collaborated on the responsibilities of this longitudinal study. Reported here are the interim results from enrollment/baseline and two additional data collection periods (waves) - one at the conclusion of the Junior 1 semester and one at the conclusion of the Junior 2 semester. While many studies have examined risk factors associated with carriage rates for MRSA in a cross-sectional format, this study sought to examine risk factors over time.

Nasal swab specimens were screened for $S$. aureus and MRSA using the standard CLS protocols and described previously. ${ }^{12,13}$ A positive MRSA swab represented an end-point for a participant. It was planned that the principal investigator would inform any participants of a positive result and that medical intervention would be offered to students by the Medical Director of the Student Health Center after a confirmatory swab was sent for testing to a certified clinical laboratory for confirmation. Treatment offered consisted of bactroban (mupirocin) for nasal decolonization and the oral antibiotic doxycycline. A swab was also planned for collection post- treatment to verify a negative nasal swab prior to the beginning of the next clinical rotation.

\section{Laboratory Analysis}

Nasal swab specimens were screened for $S$. aureus, $S$. "other," (staphylococcal species other than aureus which may be coagulase positive or negative) and MRSA using the standard media mannitol salt agar (MSA) and CHROMagar $^{\mathrm{TM}}$ MRSA screening agar (Becton Dickinson BBL, Franklin Lakes, NJ), Dry Spot Staphytect Plus test kits (Oxoid Limited, Lenexa, KS), and Dropit catalase reagent (Key Scientific Products, Round Rock, TX). Positive colony growth on CHROMagar was confirmed as MRSA by Vitek 2 (bioMérieux, Hazelwood, MO) susceptibility testing at CTMC (CTMC, San Marcos, TX) using Vitek GN19 susceptibility cards. Cards were inoculated and incubated in the Vitek 2 per manufacturer recommendations and results were analyzed by the advanced expert system, software version R04.03. All tests were performed according to the manufacturer's instructions. All growth on MSA or CHROMagar not consistent with $S$. aureus, $S$. other, or MRSA was discarded. S. aureus, MRSA, and S. epidermidis organisms were provided by Central Texas Medical Center (CTMC) as confirmed by Vitek 2 analysis and were used as positive and negative controls during inoculation of all microbiological testing.

\section{Data Screening and Analysis}

Nursing investigators entered questionnaire (Figure 1) results and CLS investigators entered laboratory results into an Excel database (Microsoft, Redmond, WA) for initial data collection. Each wave of data was verified for completeness and accuracy, and data were then pooled. Because repeated measures were nested in individuals, the primary analytical issue was within-individual clustering. In other words, an individual's own measures were likely to be more similar to each other (i.e., clustered) than they were to measurements from another individual. This positive within-individual clustering produced downwardly-biased standard error estimates and inflated test statistics. As noted elsewhere, a mixed-effects approach to analyzing longitudinal data has some key advantages over traditional repeated measures analysis of variance. ${ }^{19} \mathrm{~A}$ multilevel approach allowed for this clustering, and had more flexibility in accommodating missing cases than repeated measures analysis of variance.

An additional issue, however, was the structure of the disturbance and the possibility that the withinindividual clustering depended on time. In other words, data points closest in time were more correlated, which is consistent with autocorrelation in the form of a first-order autoregressive process, (i.e., an $\operatorname{AR}(1)$ error process). Due to the nested structure of the observations, serial correlation, and the binary-outcome dependent variables, the inves- 


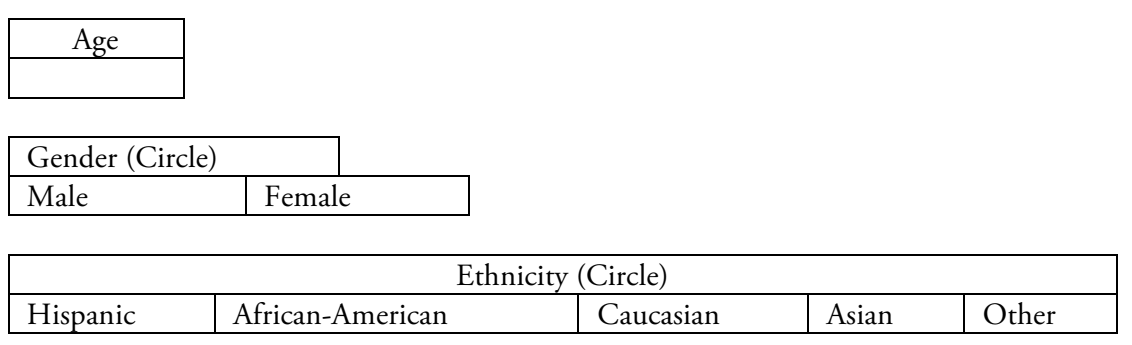

INFECTIONS:

\begin{tabular}{|c|l|l|l|l|}
\hline 1 & In the past 12 months, have you had a skin infection, boil, or sore? & Yes & No & $\begin{array}{l}\text { Don't know/ } \\
\text { Prefer not to answer }\end{array}$ \\
\hline 2 & $\begin{array}{l}\text { In the past } 12 \text { months, has a doctor told you that you have a skin infection } \\
\text { called MRSA, "mersa," or antibiotic resistant Staph? }\end{array}$ & Yes & No & $\begin{array}{l}\text { Don't know/ } \\
\text { Prefer not to answer }\end{array}$ \\
\hline 3 & Have you ever heard of MRSA, "mersa," or antibiotic resistant Staph? & Yes & No & $\begin{array}{l}\text { Don't know/ } \\
\text { Prefer not to answer }\end{array}$ \\
\hline
\end{tabular}

If so, how did you hear about it?

HEALTHCARE

\begin{tabular}{|l|l|l|l|l|}
\hline 4 & In the past 12 months, have you been a patient in the hospital? & Yes & No & $\begin{array}{l}\text { Don't know/ } \\
\text { Prefer not to answer }\end{array}$ \\
\hline 5 & In the past 12 months, have you had surgery? & Yes & No & $\begin{array}{l}\text { Don't know/ } \\
\text { Prefer not to answer }\end{array}$ \\
\hline 6 & In the past 12 months, have you worked in a healthcare facility? & Yes & No & $\begin{array}{l}\text { Don't know/ } \\
\text { Prefer not to answer }\end{array}$ \\
\hline 7 & In the past 3 months, have you taken any antibiotics? & Yes & No & $\begin{array}{l}\text { Don't know/ } \\
\text { Prefer not to answer }\end{array}$ \\
\hline 8 & In the past 12 months, have you used intravenous drugs? & Yes & No & $\begin{array}{l}\text { Don't know/ } \\
\text { Prefer not to answer }\end{array}$ \\
\hline
\end{tabular}

LIVING CONDITIONS

\begin{tabular}{|l|l|l|l|l|}
\hline 9 & Are you currently living in a dorm? & Yes & No & $\begin{array}{l}\text { Don't know/ } \\
\text { Prefer not to answer }\end{array}$ \\
\hline 10 & In the last 6 months, have you lived in a dorm? & Yes & No & $\begin{array}{l}\text { Don't know/ } \\
\text { Prefer not to answer }\end{array}$ \\
\hline 11 & In the past 12 months, have you been in jail? & Yes & No & $\begin{array}{l}\text { Don't know/ } \\
\text { Prefer not to answer }\end{array}$ \\
\hline 12 & In the past 12 months, have you participated in athletics? & Yes & No & $\begin{array}{l}\text { Don't know/ } \\
\text { Prefer not to answer }\end{array}$ \\
\hline
\end{tabular}

CLINICAL CARE EXPERIENCE- only answer for the semester you just completed.

\begin{tabular}{|c|l|l|l|l|}
\hline 13 & $\begin{array}{l}\text { During J1 semester, did you care for a patient who was on contact isolation } \\
\text { for MRSA? }\end{array}$ & Yes & No & $\begin{array}{l}\text { Don't know/ } \\
\text { Prefer not to answer }\end{array}$ \\
\hline 14 & $\begin{array}{l}\text { During J2 semester, did you care for a patient who was on contact isolation } \\
\text { for MRSA? }\end{array}$ & Yes & No & $\begin{array}{l}\text { Don't know/ } \\
\text { Prefer not to answer }\end{array}$ \\
\hline 15 & $\begin{array}{l}\text { During J3 semester, did you care for a patient who was on contact isolation } \\
\text { for MRSA? }\end{array}$ & Yes & No & $\begin{array}{l}\text { Don't know/ } \\
\text { Prefer not to answer }\end{array}$ \\
\hline 16 & $\begin{array}{l}\text { During J4 semester, did you care for a patient who was on contact isolation } \\
\text { for MRSA? }\end{array}$ & Yes & No & $\begin{array}{l}\text { Don't know/ } \\
\text { Prefer not to answer }\end{array}$ \\
\hline 17 & $\begin{array}{l}\text { During J5 semester, did you care for a patient who was on contact isolation } \\
\text { for MRSA? }\end{array}$ & Yes & No & $\begin{array}{l}\text { Don't know/ } \\
\text { Prefer not to answer }\end{array}$ \\
\hline
\end{tabular}

Figure.1. Questionnaire for risk factors to Staphylococcus aureus and Methicillin resistant Staphylococcus aureus (MRSA). 
tigators used generalized estimating equations (GEE) to obtain population-averaged panel logistic regression models. ${ }^{20}$ We also allow for an AR(1) process. Overall, analysis was conducted using Stata version 12 .

\section{RESULTS - INTERIM REPORT}

\section{Study Population}

The sample consisted of 87 nursing students over the age of eighteen, with the average age at baseline of 24.5 years. Males represented $12.6 \%$ of the sample with $74.7 \%$ of the sample Caucasian. By wave 3, 70 of the original 87 respondents provided data. The investigators found that with respect to sex and race however, attrition had not affected sample composition in any meaningful way. Furthermore, because this study will continue, we expect to recover participants in the next wave of data collection. Table 1 shows the percent of the sample testing positive for each infection across our 3 waves of data collection.
At baseline, only one respondent tested positive for MRSA. No one since has had a positive MRSA nasal swab. Similar to MRSA, the prevalence of $S$. aureus was also relatively stable over time, ranging between 20 and 26 percent. Compared to wave 1 , it was observed that a $6 \%$ increase in $S$. aureus at wave 2 occurred, but this difference was not statistically significant. The prevalence of $S$. other in this sample, however, increased steadily and relatively quickly. By the third wave of data collection, $80 \%$ of the sample tested positive for S. other, whereas less than $10 \%$ tested positive at baseline. For completeness, a difference in proportions test was utilized to compare wave 1 to wave 2 and wave 3 . As shown in Table 1, it was shown that a statistically significant increase in S. other infection occurred over time. However, we ultimately used a different estimation strategy to identify correlates of infection.

Table 1. Percent of sample with positive nasal swab

By infection type and wave

\begin{tabular}{|c|c|c|c|c|c|c|c|}
\hline & \multirow[b]{2}{*}{ Wave 1} & \multirow[b]{2}{*}{ Wave 2} & \multirow[b]{2}{*}{ Wave 3} & \multicolumn{2}{|c|}{ Wave 1 to Wave 2} & \multicolumn{2}{|c|}{ Wave 1 to Wave 3} \\
\hline & & & & Percent Change & $\mathbf{Z}$ & Percent Change & Z \\
\hline MRSA & $1.15 \%$ & $0.0 \%$ & $0.0 \%$ & NA & NA & NA & NA \\
\hline S. aureus & $20.7 \%$ & $26.4 \%$ & $20.0 \%$ & 5.7 & 0.89 & -0.7 & -0.11 \\
\hline $\begin{array}{l}S . \text { other } \\
* * *=\mathrm{p}\end{array}$ & $\begin{array}{l}9.2 \% \\
.001\end{array}$ & $68.9 \%$ & $80.0 \%$ & $59.7^{* * *}$ & 8.97 & $70.8^{* * *}$ & 8.97 \\
\hline
\end{tabular}

Note: We suggest viewing these from a descriptive statistics perspective since the alpha error rate increases with multiple comparisons.

\section{Risk Factors and Control Variables}

The central focus of this study was concerned with whether exposure to the following four conditions represented risk factors for contracting $S$. aureus, $S$. other, and MRSA. The first measure was a dummy coded item for exposure to a healthcare environment as a result of being a patient $(1=$ yes). The measure was based on answering yes to any of four relevant items; the questionnaire asked respondents whether they had (1) been a patient in a hospital; (2) had surgery; (3) taken antibiotics; or (4) used intravenous drugs since their last interview. Table 2 provides the percent of sample with exposure across waves.

The second measure was a dummy coded item also measuring exposure to a healthcare environment, but based on whether the respondent had worked or volunteered in a healthcare facility $(1=$ yes). (All students had spent a similar amount of time as nursing students.) Our third measure was whether the respondent had had close contact with someone diagnosed with MRSA since the last interview (1 = yes).

Table 2. Percent of sample with exposure to healthcare setting, people with MRSA, and gym/sports activities

\begin{tabular}{lccc}
\hline By variable and wave & Wave 1 & Wave 2 & Wave 3 \\
\hline $\begin{array}{l}\text { Exposure to healthcare settings } \\
\text { As a patient }\end{array}$ & $32.1 \%$ & $39.2 \%$ & $23.1 \%$ \\
$\begin{array}{l}\text { As a worker/volunteer } \\
\text { Contact with person }\end{array}$ & $27.5 \%$ & $78.5 \%$ & $98.5 \%$ \\
$\begin{array}{l}\text { diagnosed with MRSA } \\
\begin{array}{l}\text { Involvement in gym/ } \\
\text { sports activities }\end{array}\end{array}$ & $8.0 \%$ & $16.8 \%$ & $60.1 \%$ \\
\hline
\end{tabular}

$\mathrm{p}<.001$

Note: At baseline, the reference period is the previous 12 months. The investigators suggest viewing these from a descriptive statistics perspective since the alpha error rate increases with multiple comparisons 
The fourth measure was also a dummy coded variable based on the following two items: whether the respondent: (1) participated in athletics; or (2) used a workout center since the last interview. Such activities might independently increase the risk of contracting $S$. aureus, S. other, and MRSA. We also collected general demographic information. In our analyses, we controlled for age, sex $(1=$ male $)$, and race of the respondent (White or not-White). Additionally, we held constant whether the subject reported having a skin infection, boil, or sore since the last interview.

The first model, which explained $S$. aureus, was a relatively poor fit to the data. We observed that those who reported a boil or skin infection, compared to those who did not, had more than twice the odds of having $S$. aureus. However, the model chi-square test indicated no significant improvement over an empty model $(8.08, \mathrm{p}=.42)$. Results from the second model, which predicted testing positive for $\mathrm{S}$. other, showed two substantive findings. First, those who worked or volunteered in a healthcare facility had almost 3 times the odds of having $S$. other, which was a statistically significant difference $(\mathrm{OR}=2.72, \mathrm{p}<.05)$. Second, those who reported being involved in gym and sports activities had about 5 times the odds of having S. other, and this difference too was significant $(\mathrm{OR}=4.98, \mathrm{p}<$ .001). Results are shown in Table 3.

\section{DISCUSSION}

Since HCWs, such as nurses, work at the intersection between hospitals, healthcare facilities (e.g. dialysis units and long-term care), and nursing homes on the one hand and interact with the community (schools, athletic facilities, prisons, universities, etc.) on the other, they may serve as reservoirs, vectors, or victims of MRSA, $S$. aureus, and/or other multiple drug resistant organisms' cross-transmission. ${ }^{14}$ In this respect, HCWs have been studied extensively in a variety of individual reports with regard to sporadic, epidemic, and endemic MRSA; however, the only previous review was limited to outbreak reports. ${ }^{21}$ The investigators of this interim report set out to follow a cohort of nursing students in anticipation of exploring how staphylococci, particularly $S$. aureus and MRSA, colonize HCWs (or do not colonize) and if there is a conversion longitudinally. Most current studies and reviews of staphylococci colonization or conversion have been point prevalence studies. By documenting risks associated with the daily working routines and lifestyles of this population, this interim report highlights the findings at the halfway point of the study.

Table 3. Logistic regression models explaining positive infection

\begin{tabular}{|c|c|c|c|c|}
\hline \multirow[t]{2}{*}{ By infection type } & \multirow{2}{*}{$\begin{array}{c}\text { S. aureus } \\
\text { Odds } \\
\text { Ratio }\end{array}$} & \multicolumn{3}{|c|}{$\begin{array}{l}\text { S. other } \\
\text { Odds }\end{array}$} \\
\hline & & $\mathbf{Z}$ & Ratio & $\mathbf{Z}$ \\
\hline \multicolumn{5}{|c|}{ Exposure to healthcare settings } \\
\hline As a patient & 0.80 & -0.68 & 1.15 & 0.41 \\
\hline As a worker/volunteer & 1.42 & 1.06 & $2.72^{* *}$ & 2.95 \\
\hline $\begin{array}{l}\text { Contact with person } \\
\text { diagnosed with MRSA }\end{array}$ & 0.86 & -0.49 & 0.77 & -0.77 \\
\hline $\begin{array}{l}\text { Involvement in gym/ } \\
\text { sports activities }\end{array}$ & 1.45 & 1.13 & $4.98^{* * *}$ & 4.43 \\
\hline \multicolumn{5}{|l|}{ Control variables } \\
\hline Age & 0.99 & -0.25 & 1.01 & 0.31 \\
\hline Male & 1.13 & 0.19 & 1.40 & 0.66 \\
\hline White & 1.25 & 0.43 & 1.08 & 0.20 \\
\hline $\begin{array}{l}\text { Had boil/skin infection } \\
\text { since last interview }\end{array}$ & $2.43^{*}$ & 2.14 & 1.76 & 1.12 \\
\hline \multicolumn{2}{|l|}{ Number of observations } & 233 & & 233 \\
\hline \multicolumn{2}{|c|}{ Number of subjects } & 83 & & 83 \\
\hline \multicolumn{2}{|c|}{ Model Wald (Chi-square) } & 8.08 & & $41.75^{* * *}$ \\
\hline
\end{tabular}

${ }^{*}=\mathrm{p}<.05$

$* *=\mathrm{p}<.01$

$* * *=\mathrm{p}<.001$

The prevalence of $S$. aureus in our cohort of nursing students was slightly lower $(20-26 \%)$ but similar to previously reported studies of $19 \%$ to $37 \% .^{12,13,15-17}$ Additionally, other investigators found that age, gender, chronic sinusitis, medical student status, and hospitalization were associated with carrier status for $S$. aureus $^{15}$ which this study did not demonstrate. Surprisingly, this study found diametrically opposed findings from those of Rohde, Denham and Brannon ${ }^{13}$ in that hospitalization was not related to carrier risk, but did replicate the results of previous studies that time spent in the hospital setting as either a volunteer or healthcare worker increased this risk. Lastly, nursing students reporting a boil or skin infection during a clinical experience were almost two and a half times more likely to be colonized with $S$. aureus $(\mathrm{OR}=2.43$, $\mathrm{p}<.05)$. Skin infections have been consistently reported as a major risk factor for both MRSA and $S$. aureus colonization and infections. ${ }^{12-14}$

MRSA colonization in this nursing student cohort population did not increase and, as mentioned above, $S$.

VOL 25, NO 2 SPRING 2012 CLINICAL LABORATORY SCIENCE

99 
aureus colonization remained relatively stable. These results may have occurred because healthcare students were sensitized to risks of MRSA. At the outset of the study, nursing faculty conducted an orientation on what exactly MRSA represented and its risks in the healthcare and community environment. The orientation session may have influenced the student cohort resulting in increased compliance with use of barrier precautions (hand washing, personal protective equipment, patient handling, etc.). Indeed, nursing participants often reported "awareness" of quarantined MRSA patients and their subsequent adherence to hygiene and other infection control precautions. Many risk factors account for the increase in MRSA infections such as the overprescription of antibiotics, the emergence of new virulent strains, and healthcare-setting transmission. ${ }^{23}$ National mandates and congressional concern have erupted recently due to MRSA's high financial and human costs, not to mention other HAIs. Hospitals are being asked to develop aggressive protocols to decrease HAIs such as MRSA colonization and infection. ${ }^{24}$ There are many approaches being followed including the use of MRSA prevention bundles in surgical site infections. ${ }^{25}$ In almost all approaches, education and compliance play a part of a critical, if not the singular important role, in the decrease of MRSA colonization and infection. The investigators of this study believe that our initial orientation and continued focus on hygiene, fomites, reservoirs, and prevention-minded thinking had an impact on the interim MRSA colonization of our nursing students remaining at a zero level.

An unexpected finding at the interim point of this investigation is the nasal colonization of participants with species other than $S$. aureus (e.g. S. epidermidis, S. intermedius, $S$. haemolyticus, etc.). The prevalence of $S$. other (species other than aureus) in this sample increased steadily and relatively quickly. The investigators chose to use "S. other" in this study instead of coagulase negative staphylococci (CNS) because there are species other than $S$. aureus that can be coagulase positive (e.g. S. delphini, S. hyicus, S. intermedius, S. lutrae, S. pseudintermedius, S. schleiferi subsp. Coagulans and S. leei) as well as the CNS. By the third wave of data collection, $80 \%$ of the sample tested positive for S. other, whereas less than $10 \%$ tested positive at baseline. Participants who worked or volunteered in a healthcare facility had almost three times the odds of having $S$. other, while those who reported being involved in gym and sports activities had about five times the odds of having $S$. other, and this difference too was significant $(\mathrm{OR}=4.98, \mathrm{p}<.001)$. While other studies have shown that being involved with a healthcare facility and athletics are risk factors for MRSA and/or $S$. aureus, ${ }^{2-4,7,12,13,15}$ it has rarely or never been reported with species other than $S$. aureus nor has the rapid increase in prevalence. The investigators hypothesize that this may be explained simply by more participants using indoor workout facilities (e.g. gyms, recreational centers, etc.) more during the winter versus the summer when specimens were first collected. It is not well-understood if this phenomenon may play an important role in nosocomial transmission understanding. Perhaps, colonization with other staphylococci species may play a protective role by competitive inhibition of other pathogens in nasal colonization, or conversely, perhaps it may play a role in HAIs that have not been documented. The investigators will follow this unexpected finding through the completion of the study and anticipate gaining additional insight by determining the types of different staphylococci species present.

\section{CONCLUSION}

MRSA, along with other HAIs, has emerged as a growing world-wide problem in the past decade(s). Common-sense approaches to prevention, along with intelligent use of the laboratory (culture of wounds, antibiotic susceptibility testing, etc.) and available antimicrobials can protect individuals from this new threat. Healthcare officials, community leaders, and public health policy-makers should be aware of the potential transmission risk and outbreak scenario that could develop in the rich environment of HCW populations and their daily work-related tasks. This study, along with others referenced, illustrates the growing importance of patient and community education and where it intersects with compliance of basic infection control prevention efforts. Finally, research is desperately needed in the area of knowledge, awareness, and the learning needs (gaps in knowledge) of the general public with respect for MRSA and other antibiotic resistant organisms. ${ }^{22}$

\section{ACKNOWLEDGEMENTS}

Thanks are expressed to Dean Ruth Welborn PhD RN, Texas State University, College of Health Professions for financial and infrastructure support of this study. 
Additionally, appreciation to CLS (Carmen Adams $\mathrm{PhD}$, Dave Falleur MEd MT, students at time of study - Stephen Garcia, Jacella McKenzie, Stephanie Jimenez MT, Emma Straten MT, Cindy Diaz, Terri Davis and Kayla Cross) and the School of Nursing (Director, Professor and Associate Dean, Marla Erbin-Roesemann $\mathrm{PhD} \mathrm{RN}$; Barbara G. Covington, PhD RN; and Rita Zapata Mokarzel) at Texas State University for their help and time with laboratory work, collection of data, and oversight of materials. Additionally, a special thanks to Nathalie Austin MBA MT (TX State alumni) at Central Texas Medical Center for providing confirmation of isolates.

\section{REFERENCES}

1. Centers for Disease and Prevention. MRSA surveillance. Available from http://www.cdc.gov/mrsa/statistics/MRSASurveillance-Summary.html. Accessed 9/16/2011

2. Klevens RM, Morrison MA, Nadle J, et al. Invasive methicillin-resistant Staphylococcus aureus infections in the United States. JAMA 2007;298(15):1763-71.

3. Kenner J, O'Connor T, Piantanida N, et al. Rates of carriage of methicillin-resistant and methicillin-susceptible Staphylococcus aureus in an outpatient population. Infect Control Hosp Epidemiol 2003;24(6):439-44.

4. Weiner, R. Methicillin-resistant Staphylococcus aureus on campus: A new challenge to college health. J American College Health 2008;56(4):347-50.

5. Banning M. Transmission and epidemiology of MRSA: Current perspectives. Br J Nurs 2005;14(10):548.

6. Chi C, Wong W, Fung C, et al. Epidemiology of communityacquired Staphylococcus aureus bacteremia. J Microbiol Immunol Infect 2004;02;37(1):16-23.

7. Beam JW, Buckley B. Community-acquired methicillinresistant Staphylococcus aureus: Prevalence and risk factors. J Athl Train 2006;07;41(3):337-40.

8. Mainous AG, Hueston WJ, Everett CJ, et al. Nasal carriage of Staphylococcus aureus and methicillin-resistant $S$. aureus in the United States, 2001-2002. Ann Fam Med 2006;03;4(2):132-7.

9. Huang $\mathrm{H}$, Cohen $\mathrm{SH}$, King $\mathrm{JH}$, et al. Injecting drug use and community-associated methicillin-resistant Staphylococcus aureus infection. Diagn Microbiol Infect Dis 2008;60(4):34750 .

10. Turabelidze G, Lin M, Wolkoff B, et al. Personal hygiene and methicillin-resistant Staphylococcus aureus infection. Emerg Infect Dis 2006;12(3):422-7.

11. Moran GJ, Krishnadasan A, Gorwitz RJ, et al. Methicillin- resistant $S$. aureus infections among patients in the emergency department. N Engl J Med 2006;355(7):666-74.

12. Felkner M, Rohde RE, Valle-Rivera AM, et al. Methicillinresistant Staphylococcus aureus nasal carriage rate in Texas county jail inmates. J Correctional Health Care 2007;13(4):289-95.

13. Rohde RE, Denham R, Brannon A. Methicillin Resistant Staphylococcus aureus: Nasal carriage rate and characterization in a Texas university setting. Clin Lab Sci 2009;22(3):176-84.

14. Albrich WC, Harbath S. Health-care workers: source, vector, or victim. Lancet, Infection Dis 2008;8:289-301.

15. Bischoff WE, Wallis ML, Tuckert KB, et al. Staphylococcus aureus nasal carriage in a student community: Prevalence, clonal relationships, and risk factors. Infection Control and Hospital Epidemiology 2004;25:485-91.

16. Stubbs E, Pegler M, Vickery A, et al. Nasal carriage of Staphylococcus aureus Australian (pre-clinical and clinical) medical students. J Hosp Infect 1994;27:127-34.

17. Kingdom JC, Joyce SM, Bradley FL, et al. Staphylococcal nasal carriage in medical students with varying clinical exposure. J Hosp Infect 1983;4:75-9.

18. Dunkelberg H. On the incidence of Staphylococcus aureus in the throat of medical students. Zentralbl Bakteriol 1976;163:530-5.

19. Wray LO, Shulan MD, Toseland RW, Freeman K, et al. The effect of telephone support groups on costs of care for veterans with dementia. The Gerontologist 2010;50:623-31.

20. Zeger SL, Kung-Yee, L. Longitudinal data analysis for discrete and continuous outcomes. Biometrics 1986;42:121-30.

21. Vonberg RP, Stamm-Balderjahn S, Hansen $S$, et al. How often do asymptomatic healthcare workers cause methicillin-resistant Staphylococcus aureus outbreaks? A systematic evaluation. Infect Control Hosp Epidemiol 2006;27:1123-7.

22. Rohde RE. Methicillin resistant Staphylococcus aureus (MRSA) knowledge, learning, and adaptation: I guess everything changes when it happens to you - their stories. Saarbrücken, Germany: Lambert Academic Publishing GmbH \& Co. KG; 2011.

23. Griffin FA, Reducing methicillin-resistant Staphylococcus aureus infection. Joint Comm Accreditation Healthcare Organ 2007;33:12.

24. Institute for Health Care Improvements (IHI). 5 millions lives campaign, reduce methicillin-resistant Staphylococcus aureus infection. Available from http://www.IHI.org. Accessed 9/10/2011.

25. Awad SS, Palacio CH, Subramanian A, et al. Implementation of a methicillin-resistant Staphylococcus aureus (MRSA) prevention bundle results in decreased MRSA surgical site infections. The Am J of Surg 2009;198(5):607-10. 